\title{
The transformation of earth-system observations into information of socio- economic value in GEOSS
}

Article

Published Version

Hollingsworth, A., Uppala, S., Klinker, E., Burridge, D., Vitart, F., Onvlee, J., De Vries, J. W., De Roo, A. and Pfrang, C. (2005) The transformation of earth-system observations into information of socio-economic value in GEOSS. Quarterly Journal of the Royal Meteorological Society, 131 (613). pp. 3493-3512. ISSN 1477-870X doi:

https://doi.org/10.1256/qj.05.181 Available at https://centaur.reading.ac.uk/17082/

It is advisable to refer to the publisher's version if you intend to cite from the work. See Guidance on citing.

To link to this article DOI: http://dx.doi.org/10.1256/qj.05.181

Publisher: Royal Meteorological Society

All outputs in CentAUR are protected by Intellectual Property Rights law, including copyright law. Copyright and IPR is retained by the creators or other copyright holders. Terms and conditions for use of this material are defined in the End User Agreement. 


\section{CentAUR}

Central Archive at the University of Reading

Reading's research outputs online 


\title{
The transformation of earth-system observations into information of socio-economic value in GEOSS
}

\author{
By ANTHONY HOLLINGSWORTH ${ }^{1 *}$, SAKARI UPPALA ${ }^{1}$, ERNST KLINKER ${ }^{1}$, DAVID BURRIDGE ${ }^{1}$, \\ FREDERIC VITART $^{1}$, JEANETTE ONVLEE ${ }^{2}$, J. W. DE VRIES ${ }^{2}$, AD DE ROO $^{3}$ and CHRISTIAN PFRANG ${ }^{1}$ \\ ${ }^{1}$ European Centre for Medium-Range Weather Forecasts, Reading, UK \\ ${ }^{2}$ Koninklijk Nederlands Meteorologisch Instituut, De Bilt, the Netherlands \\ ${ }^{3}$ European Union Joint Research Centre, Institute for Environment and Sustainability, Ispra, Italy
}

(Received 26 August 2005; revised 1 March 2006)

\section{SUMMARY}

The Group on Earth Observations System of Systems, GEOSS, is a co-ordinated initiative by many nations to address the needs for earth-system information expressed by the 2002 World Summit on Sustainable Development. We discuss the role of earth-system modelling and data assimilation in transforming earth-system observations into the predictive and status-assessment products required by GEOSS, across many areas of socio-economic interest. First we review recent gains in the predictive skill of operational global earth-system models, on timescales of days to several seasons. We then discuss recent work to develop from the global predictions a diverse set of end-user applications which can meet GEOSS requirements for information of socio-economic benefit; examples include forecasts of coastal storm surges, floods in large river basins, seasonal crop yield forecasts and seasonal lead-time alerts for malaria epidemics. We note ongoing efforts to extend operational earth-system modelling and assimilation capabilities to atmospheric composition, in support of improved services for air-quality forecasts and for treaty assessment. We next sketch likely GEOSS observational requirements in the coming decades. In concluding, we reflect on the cost of earth observations relative to the modest cost of transforming the observations into information of socio-economic value.

KEYWORDS: Earth-system models Lead-time alerts Multi-seasonal forecasting

\section{INTRODUCTION}

Following the adoption of the Johannesburg Plan of Implementation by the 2002 World Summit on Sustainable Development, in 2003 ministers from many nations plus the European Commission adopted a Declaration of political commitment to move toward development of a comprehensive, co-ordinated and sustained earth-observation system, and affirmed the need for timely, high-quality, long-term, global information as a basis for sound decision making. In order to monitor continuously the state of the earth, to increase understanding of dynamic earth processes, to enhance prediction of the earth system, and to further implement environmental treaty obligations, the adherents to the Declaration recognized the need to support the creation of a comprehensive, co-ordinated, and sustained earth-observing system of systems. This initiative, known as GEOSS (Group on Earth Observations System of Systems), developed a 10-year Implementation Plan (GEOSS 2005) which was formally adopted by some 60 nations in 2005; the nations are supported in the GEOSS undertaking by about 40 international organizations having a mandate in earth observations.

In the words of the GEOSS Framework Document (GEOSS 2004):

"Understanding the earth system-its weather, climate, oceans, land, geology, natural resources, ecosystems, and natural and human-induced hazards - is crucial to enhancing human health, safety and welfare, alleviating human suffering including poverty, protecting the global environment, and achieving sustainable development. Data collected and information created from earth observations constitute critical input for advancing

\footnotetext{
* Corresponding author: European Centre for Medium Range Weather Forecasts, Shinfield Park, Reading,
} Berkshire RG2 9AX, UK. e-mail: a.hollingsworth@ecmwf.int

(C) Royal Meteorological Society, 2005. 
this understanding. Comprehensive, co-ordinated and sustained earth observations for understanding the earth system more completely and comprehensively will expand worldwide capacity and means to achieve sustainable development, and will yield advances in many specific areas of socioeconomic benefit, including:

- $\quad$ Reducing loss of life and property from natural and human-induced disasters;

- Understanding environmental factors affecting human health and well being;

- Improving management of energy resources;

- Understanding, assessing, predicting, mitigating, and adapting to climate variability and change;

- Improving water resource management through better understanding of the water cycle;

- Improving weather information, forecasting, and warning;

- Improving the management and protection of terrestrial, coastal, and marine ecosystems;

- $\quad$ Supporting sustainable agriculture and combating desertification;

- Understanding, monitoring, and conserving biodiversity".

The European initiative on Global Monitoring for Environment and Security (GMES, www.gmes.info), which began in 1998, will be the main European contribution to GEOSS.

The GEOSS and GMES agendas have substantial implications for the development of earth-system modelling and data assimilation capabilities. In section 2 we describe how a 'cascade' of earth-system modelling and assimilation capabilities can transform earth-system observations into the predictive and status-assessment products required by GEOSS and GMES. After a review of gains in predictive skill in operational global systems (on time-scales of a few days to several seasons), section 3 discusses diverse recent developments in each of which a cascade of suitable earth-system models provides a set of products of direct relevance to the GEOSS areas of socio-economic benefit; these include: forecasts of coastal storm surges, floods in large river basins, energy pricing, seasonal forecasts of crop yields, and seasonal lead-time alerts of epidemic malaria. We also note current efforts to extend operational earth-system modelling and assimilation capabilities to atmospheric composition, in order to provide new deliverables in the climate, air-quality and health sectors. The likely GEOSS observational requirements in coming decades are discussed in section 4 . We conclude in section 5 with some reflections on the high cost of earth observations relative to the modest costs of transforming the observations into information of socio-economic value.

\section{TRANSFORMATION OF EARTH-SYSTEM OBSERVATIONS INTO PRODUCTS OF SOCIO-ECONOMIC VALUE}

Governments, through the GEOSS initiative, are demanding much more than science alone from their investments in earth-system observations and from the scientists they fund to use those observations. Governments realize that with better organization and co-ordination, they can realize a wide range of socio-economic benefits in the management and development of the weather-sensitive, climate-sensitive and environmentsensitive sectors of society. The GEOSS Implementation Plan (GEOSS 2005) aims 

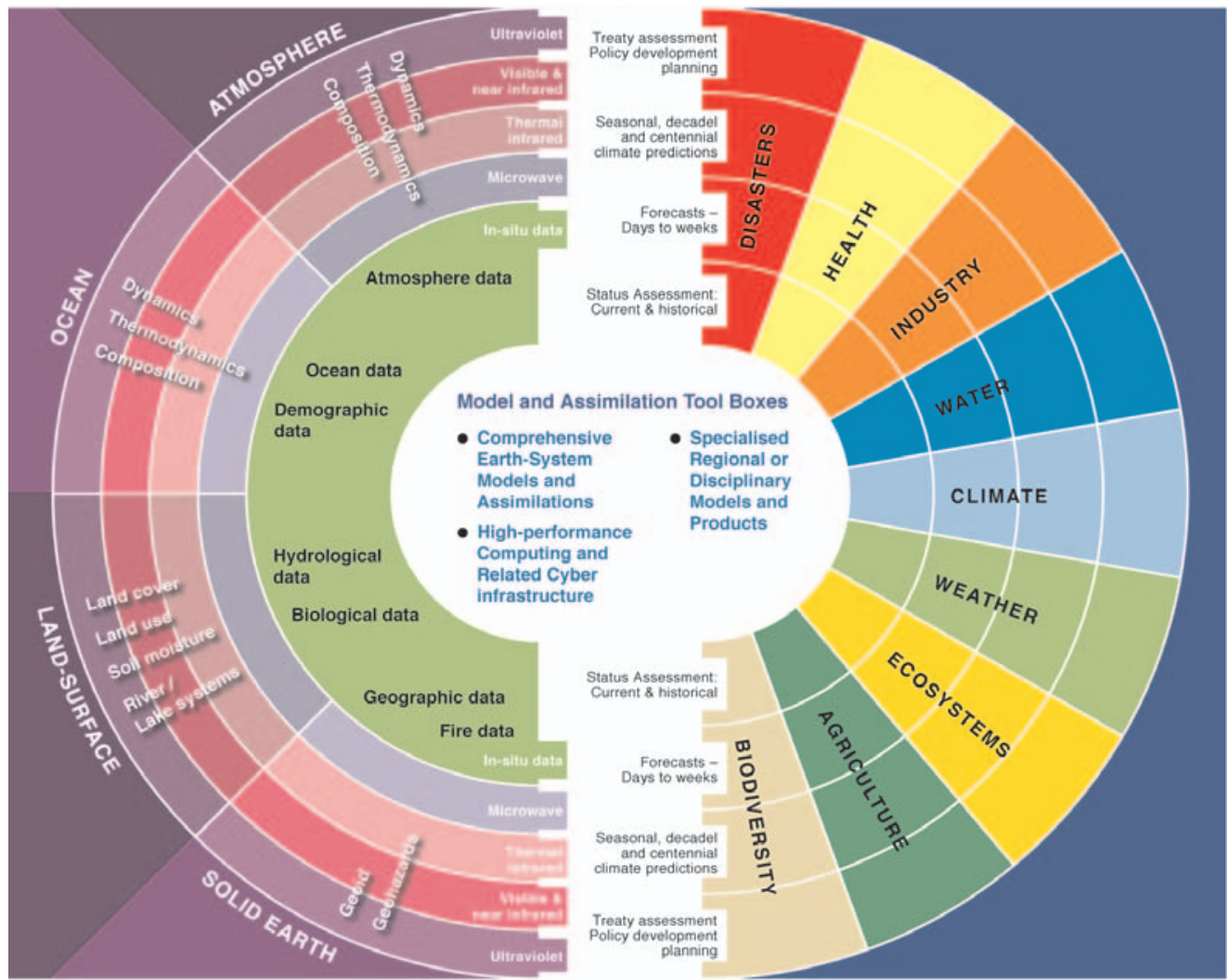

Figure 1. Based on the Group on Earth Observations System of Systems (GEOSS) Implementation Plan, a schematic illustration of the transformation of observations into predictive and current-status information. On the right-hand side: deliverables from an earth-system forecasting system and associated specialized models organized in the GEOSS categories of socio-economic benefits, stratified by the lead-time on which the deliverables are required (current status assessments, forecast time-range, long-term studies of re-analysis). On the lefthand side: observational requirements for a comprehensive earth-system model, including in situ data plus current and projected satellite data (both operational and research satellites). Many space-based requirements are met by current operational and research missions, other requirements are unfulfilled. The satellite missions are categorized by the geophysical domain observed (atmosphere, ocean, land-surface, solid earth) and by the electromagnetic frequency used by the missions. In the centre: tool-boxes needed at an earth-system forecasting centre (e.g. ECMWF) and at specialized customer/associate sites, to achieve the transformation from observations to information.

to deliver socio-economic benefits across a wide variety of domains and time-scales, through improved exchange and exploitation of a wide array of in situ and space-based observations of the earth system. The GEOSS deliverables may be categorized in two broad categories-current status-assessments and predictions. Earth-system modelling and data assimilation methods will play a central role in delivering both categories of products.

The schematic in Fig. 1 is designed to suggest the ambition, complexity and the feasibility of the GEOSS objectives. The 'observational' (left-hand) side of Fig. 1 sketches the diverse in situ (green) and satellite data sources (arranged by electromagnetic frequency used) needed to achieve the diverse GEOSS goals; it is instructive to populate the observational side of the diagram with the many existing and planned in situ and satellite observing systems, including both research and operational satellite systems. 
The 'deliverable' (right-hand) side of the figure sketches the diversity of the GEOSS deliverables, organized in relation to the GEOSS areas of socio-economic benefit, and stratified by the time-scale for which the deliverables are relevant.

The centre of the diagram summarizes the process used to transform the observations on the left of the figure into the information products on the right (both predictive and status-assessment products). The process involves a cascade or hierarchy of models and data assimilation systems. The first stage of the cascade (in the lefthand semi-circle) involves complex earth-system models and data assimilation systems (e.g. coupled atmosphere-ocean-land-cryosphere-biosphere models) which synthesize a very wide range of observations to make comprehensive global status assessments and predictions; such systems usually require substantial computer resources. In the second stage of the cascade (right-hand semi-circle) the outputs of the comprehensive global models drive a range of specialized application models which refine the global information to the specific information needs of regional and local users, in domains as diverse as health, energy, water resources, coastal protection, crop yields, air quality etc. The outputs of the specialized models are increasingly exploited in decision aids such as geographic information systems.

In November 2004 we made an informal assessment of the development of GEOSSrelevant applications, through an internet survey of such developments which use data from the European Centre for Medium-Range Forecasts (ECMWF) 45-year reanalysis (ERA-40; Uppala et al. 2005). The ERA-40 datasets are re-analysed gridded global meteorological and surface fields available four times daily for the period from September 1957 to August 2002. Google was employed as the search engine using a large number of keywords like 'flood' or 'greenhouse gases' in conjunction with the 'ERA40' keyword. The web hits were categorized into activity areas and time-scales of forecasts according to the categories on the right-hand side of Fig. 1. A 'clickable' image-map of the right-hand side of the figure was linked to a table containing all the information retrieved in the internet survey, to provide an overview of research areas of ERA-40 applications relevant to GEOSS*. The results show a wide range of activity underway around the world that can be harnessed to meet the GEOSS deliverables.

\section{INFORMATION PRODUCTS DELIVERED THROUGH A CASCADE OF EARTH-SYSTEM MODELS}

The sustained availability of earth-system observations over recent decades has stimulated scientific efforts to understand and model a broad range of earth-system processes, and thus to deliver useful predictions and status-assessments. In this section, after a review of recent gains in predictive skill on medium-range time-scales (310 days), weekly to monthly time-scales, and multi-seasonal time-scales, we show examples of a variety of forecasts and status-assessments of direct socio-economic benefit, generated using the output of comprehensive earth-system models to drive specialized models which deliver tailored information products to end-users.

\section{(a) Developments in medium-range forecasting models and ensemble methods}

The 1980-2005 record of annual-average operational weather-forecast skill for the northern and southern hemispheres is shown in Fig. 2(a), where skill is measured by the anomaly correlation of the $500 \mathrm{hPa}$ forecasts at 3-, 5- and 7-day lead times; this figure is an updated version of Fig. 4 in Simmons and Hollingsworth (2002) and of Fig. 14 in

\footnotetext{
* See http://www.ecmwf.int/research/era/era40survey/
} 
Uppala et al. (2005). The operational scores show sustained gains of about 1 day per decade in forecast skill in both hemispheres since 1980. Since about 1999 the skill for the southern hemisphere is almost as high as that for the northern hemisphere. Simmons and Hollingsworth (2002) showed that the gains could be attributed to improvements in: the science of modelling and data assimilation, observations, and the computing resources which determine model resolution. It is not easy to separate the impact of the individual contributions to forecast skill because of the central role played by the model in the data assimilation process.

The operational system that produced the forecasts in Fig. 2(a) was in continuous evolution throughout the period. Uppala et al. (2005) describe the ERA-40 re-analysis system and the re-analysed products covering the period September 1957 to August 2002; the ERA-40 system corresponds qualitatively to the operational systems used in the second half of the 1990s. Using the invariant ERA-40 forecast system on the daily data between 1957 and 2002, Uppala et al. (2005) show that a fundamental improvement in the observing system occurred in the late 1970s, almost certainly from the introduction of the TIROS* Operational Vertical Sounder (TOVS) and geostationary satellite observing systems, although there were changes in the in situ observing system at the same time. Figure 2(b), reproduced from Uppala et al. (2005), shows that for the period 1980-2002 the forecast performance with the invariant ERA-40 system (strictly, one should speak of the hindcast performance) is much more uniform in time than the performance of the real-time operational system. The implication is that the substantial improvements in operational skill since 1979 are due mainly to the developments in the science of modelling and data assimilation since that time, and only partly to improvements in the satellite observing system, although caveats are noted by Uppala et al. (2005). The challenge of understanding the information in the satellite measurements has been a major stimulus to scientific developments, so the satellite contribution to current forecast performance comes partly from the stimulus to science over recent decades and partly from the information on initial conditions, whose accuracy is essential for forecast skill. The fact that since 1999 the operational southern hemisphere forecasts have been almost as good as those for the northern hemisphere could not have been achieved without the satellite data, but it took 20 years to develop the science and the computational technology to exploit effectively the observing systems put in place in the late 1970s.

A central challenge in numerical weather prediction (NWP) is the modelling and assimilation of the hydrological cycle, especially by using remotely sensed data affected by rain and clouds. This challenge poses formidable research problems- the 2005 extension of ECMWF's operational four-dimensional variational system to the assimilation of rain-affected microwave radiances (Andersson et al. 2005) required a substantial and sustained research effort over 8 years. Given the central role of the hydrological cycle in the atmosphere, there is every reason to expect that improved models and assimilation systems, together with a sustained flow of new high-quality observations, will yield steadily improving forecasts. It is worth noting that the cumulative scientific experience of recent years has shortened the time between launch of a research satellite and the transition to operational use of the data from the satellite. McNally et al. (2006) report a period of 18 months between the May 2002 launch of the AQUA mission of NASA (the National Aeronautics and Space Administration), and the October 2003 initiation of operational assimilation of the data from an entirely new type of instrument, the Advanced Infrared Sounder (AIRS), on AQUA.

\footnotetext{
* Television Infrared Observation Satellite.
} 

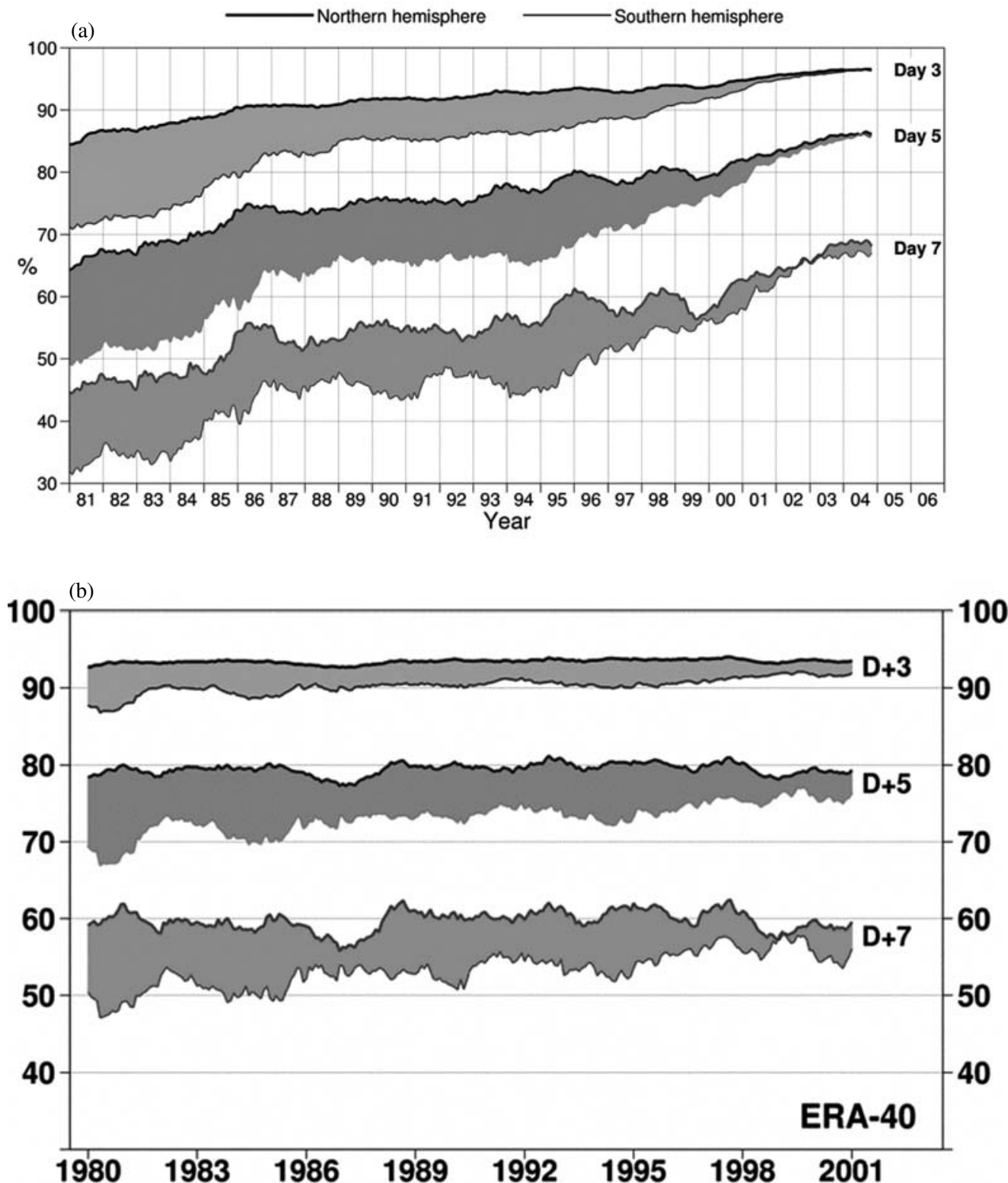

Figure 2. Twelve-month running mean anomaly correlations (\%) of 3-, 5- and 7-day 500 hPa height forecasts for the extratropical northern (bold lines) and southern (thin lines) hemispheres: (a) operational 12 UTC ECMWF forecasts from January 1980 to April 2005; (b) 12 UTC ERA-40 (see text) forecasts from January 1980 to August 2002. The shading shows the difference in scores between the two hemispheres at the forecast ranges indicated.

(From Simmons and Hollingsworth 2002, and Uppala et al. 2005.)

The last decade has seen important developments in the use of ensemble forecasts to estimate forecast uncertainty (Buizza et al. 2005). Generally, the model used to generate the ensembles has lower resolution (and less cost per single forecast) than the resolution of the model used for the deterministic forecasts. Initial data for the ensemble forecasts are perturbed in order to sample the uncertainties in the starting data, and the forecast model includes a stochastic element to represent the inevitable stochastic events on 
the subgrid-scale. The ensemble forecasts provide a good basis for the formulation of probabilistic forecasts.

A key further development has been the coupling of weather model output to enduser models, to generate ensembles which estimate the uncertainty in forecasts of enduser variables - this is the 'cascade process' described in section 2, and it can be used for both deterministic forecasts and for ensemble forecasts. One such example is the use of the method for forecasting electricity demand (Taylor and Buizza 2003). The following examples provide further illustrations of the process.

(i) Hindcasts of the Dutch storm surge of 1 February 1953. On 31 January and 1 February 1953 an intense storm traversed the Irish Sea, Great Britain and the North Sea, creating severe devastation - the ferry Princess Victoria foundered in the Irish Sea with the loss of about 132 lives, and the storm surge in the North Sea overwhelmed coastal defences in England and the Netherlands, killing some 307 persons along the English coast and some 1835 persons along the coast of the Netherlands. No effective warnings of the inundations were provided to the civil population of either country. The proceedings of a commemorative symposium provide much more detail*.

Figure 3 shows two sets of hindcasts of the sea-level at the port of Vlissingen, as well as observations; the hindcasts have lead times of 36 and $108 \mathrm{~h}$ before 0000 UTC on 1 February 1953, the peak of the storm. The starting data for the forecasts were created by assimilating the observations for the relevant period using the ERA40 system. Deterministic weather forecasts were made at a resolution of $\sim 40 \mathrm{~km}$ (T511), and 51-member ensemble weather forecasts were made at a resolution of $\sim 120 \mathrm{~km}$ (T159). The KNMI $\dagger$ operational storm surge model (developed by the Dutch Rijkswaterstaat; Gerritsen et al. (1995)) used these weather hindcasts to generate the storm-surge hindcasts shown in the figure. The critical water level for the sea defences at Vlissingen was $3.7 \mathrm{~m}$, and the observations show the surge peaking at about $4.3 \mathrm{~m}$. The observations used in the hindcasts were those available in 1953. Had those hindcasts been available in 1953, it is clear that there would have been alerts for a severe event at 4-5 days lead, and the accuracy of the alerts would have improved as the lead time shortened. Given the improved modern observing system, one would expect even better warnings to be possible today.

The quality of the hindcasts is attributable to the observations available in 1953, and to scientific and computing capabilities developed since 1953. Because of the cost of observations, forecasters are continually pressed to demonstrate the value of their observations in terms understandable to the general public. The present casestudy suggests that hindcast studies of major disasters of the past can provide dramatic illustrations of the benefit of earth observations, science and forecasts.

(ii) European flood alert system. In recent years, Europe has been much affected by devastating floods in large trans-national river basins. The benefits of coupling weather models to distributed hydrological models to generate medium-range ensemble flood alerts was assessed by De Roo et al. (2003). Figure 4 shows an example of such an ensemble hindcast for the Meuse flood of January 1995, made by coupling the ECMWF meteorological model to a distributed hydrological model (LISFLOOD). The figure shows the observed discharge at the Borgharen gauging station on the River Meuse, Netherlands, for a period of 20 days from 0000 UTC 19 January 1995 (thick line). It also shows 10-day deterministic forecasts of the discharge (from 0000 UTC 21 January 1995,

\footnotetext{
* See http://www.knmi.nl/kenniscentrum/watersnood_symposium.html

$\dagger$ Koninklijk Nederlands Meteorologisch Instituut.
} 


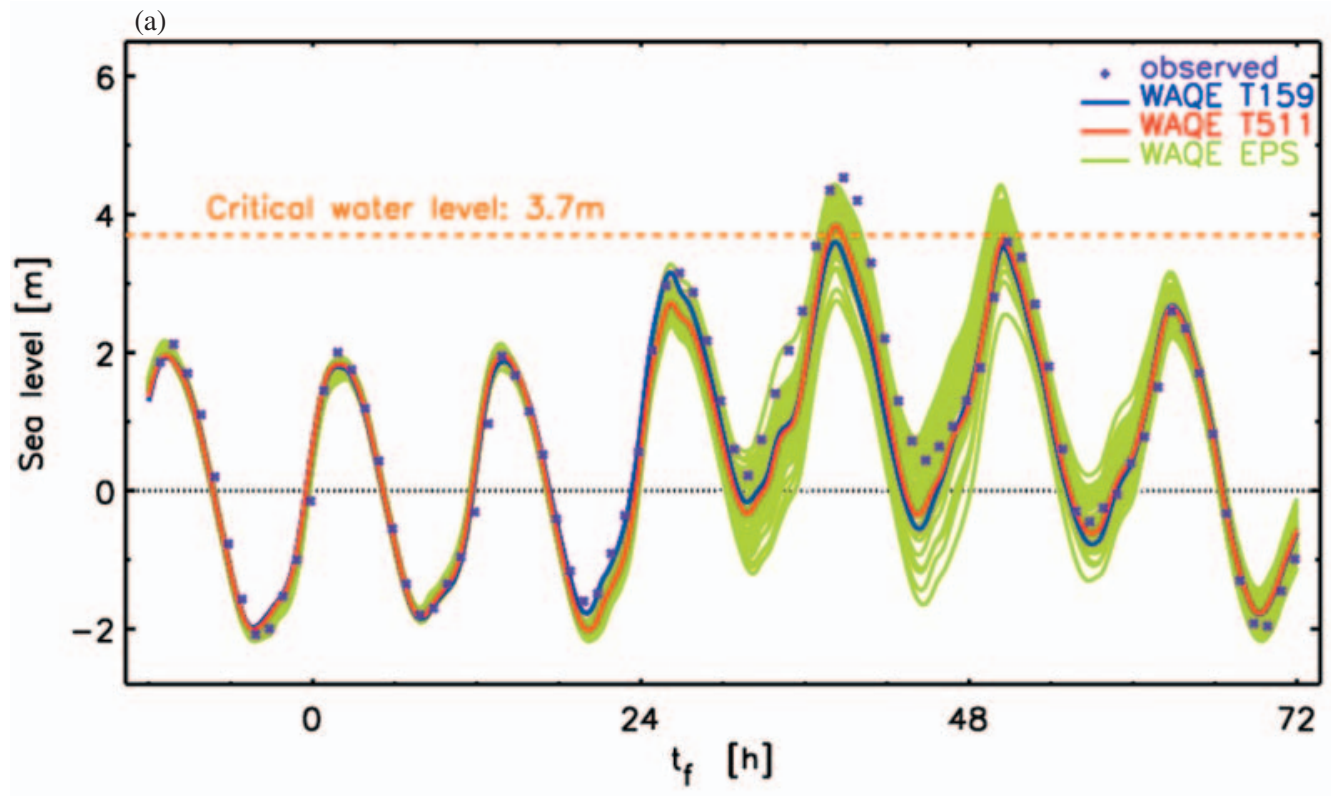

(b)

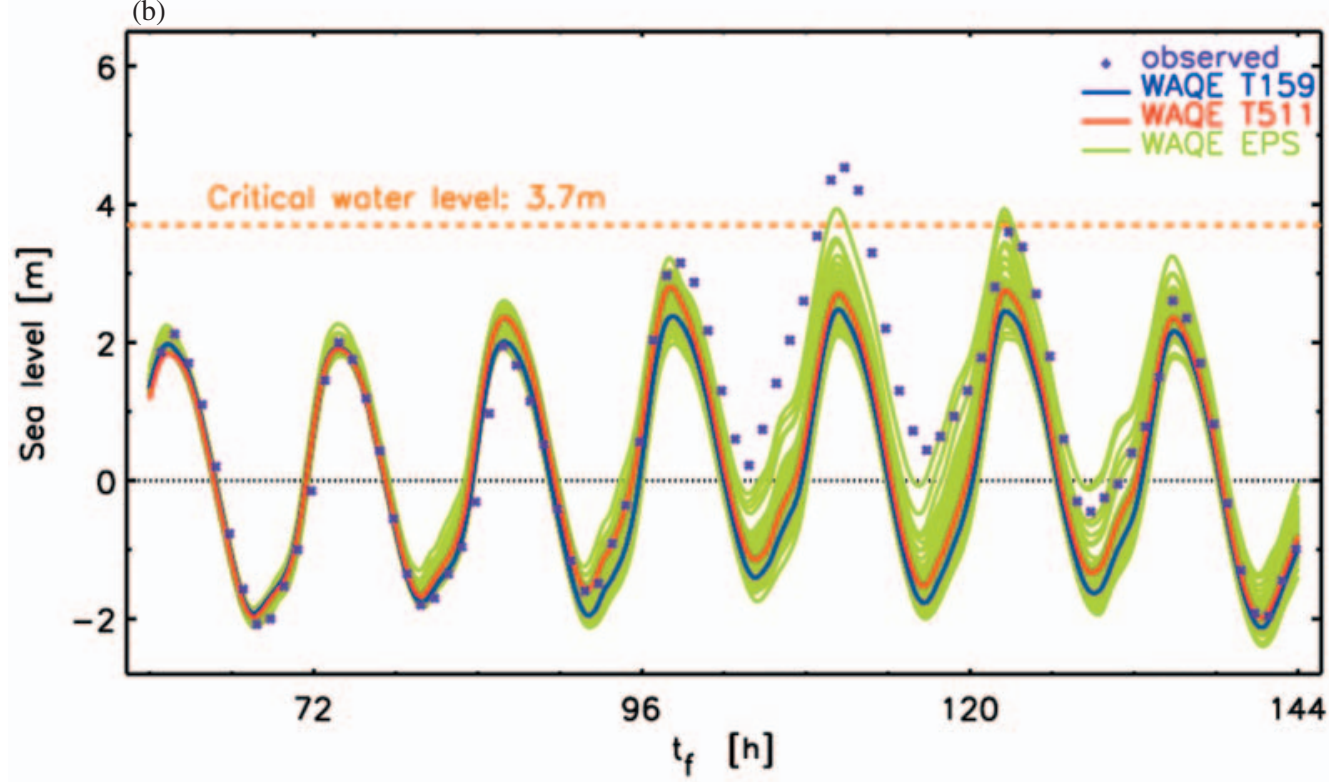

Figure 3. Hourly sea-levels observed at Vlissingen (dots), peaking at 0000 UTC on 1 February 1953, together with a series of sea-level forecasts made with the KNMI storm-surge model, forced by ECMWF weather forecasts run at T159 $(125 \mathrm{~km})$ resolution (blue), T511 $(40 \mathrm{~km})$ resolution (red), and in ensemble mode $(\mathrm{N}=51, \mathrm{~T} 159$, green), all starting from re-analyses with the ERA-40 system. The critical sea-level for over-topping of the dykes at Vlissingen was $3.7 \mathrm{~m}$ (orange). The forecasts were started: (a) 36 hours (1.5 days), and (b) 108 hours (4.5 days) before the peak of the storm-surge. See text for details. 


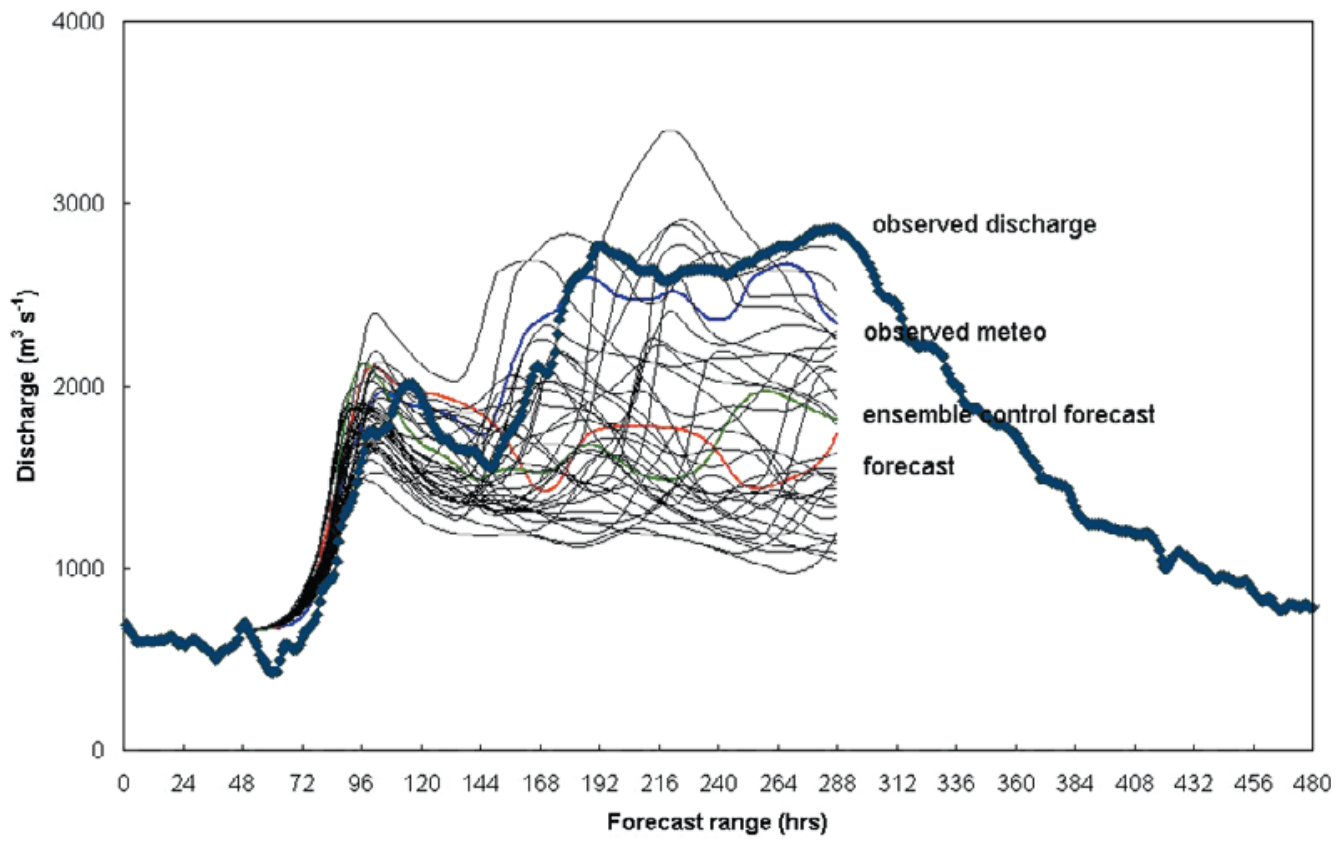

Figure 4. The observed discharge (thick line) for a 20-day period (0000 UTC 19 January 1995 to 0000 UTC 8 February 1995) for the Borgharen gauging station on the River Meuse, in the Netherlands; during this period there was a major flood on the Meuse. The plot also shows forecast simulations (hindcasts) of discharge at Borgharen, made with a distributed hydrological model (LISFLOOD) driven by different versions of the ECMWF forecast system. The simulation driven by observed meteorological data is shown as a thin blue line; the simulation driven by the $40 \mathrm{~km}$ (T511 L60) ECMWF deterministic forecast is shown in red; the simulation driven by the ECMWF $80 \mathrm{~km}$ (TL255 L40) ensemble control forecast is shown in green; and the simulations driven by the 50 ECMWF TL255L40 ensemble forecast members are shown in black. All the forecasts started from 0000 UTC on 21 Jan 1995 (labelled hour 48), and were run for 10 days. Most of the discharge forecasts are quite good for 5 days, capturing the initial rise and small decline of the discharge. In addition many of the ensemble members provide an alert for a further rise in the discharge with a lead of 5-7 days. (From de Roo et al. 2003.)

labelled $48 \mathrm{~h}$ on the time axis) where the distributed hydrological model is driven by single meteorological forecasts from the $40 \mathrm{~km}$ (TL511/L60) and $80 \mathrm{~km}$ (TL255/L40) versions of the ECMWF model. In addition an ensemble of discharge forecasts is shown, driven by the 50-member meteorological ensemble; finally the discharge calculated using the observed precipitation in the basin is shown for comparison. The coupled hindcast simulations show that the coupled hydro-meteorological system provides a good forecast of discharge up to 5 days ahead and a probabilistic assessment of extreme flooding for forecast lead times in the range 5-10 days. Further examples of successful medium-range forecasts, for extensive floods in the Po valley, may be found in Hollingsworth et al. (2004).

These and related studies (Arduino et al. 2005; Gouweleeuw et al. 2005; Pappenberger et al. 2005) have led to the implementation of a pre-operational coupled hydro-meteorological forecast system for medium-range flood alerts in large transnational river basins in Europe, known as the European Flood Alert System (EFAS; http://efas.jrc.it/.). The pre-operational EFAS system involves collaboration between the EU Joint Research Centre in Italy responsible for running the distributed hydrological model, meteorological institutes which provide the global and regional real-time meteorological forecasts (e.g. ECMWF, Deutsches Wetterdienst), and National Hydrological Services in a growing number of large European basins. The pre-operational 
phase is expected to run for several years to assess real-time experience in a variety of hydro-meteorological situations.

(iii) Soil moisture: a synergy between NWP and high-precision satellite gravimetry. The use of data assimilation systems to provide effective long-term monitoring of the quality and availability of observations has been well-established for several decades (Hollingsworth et al. 1986) and is now an important part of the routine work of NWP centres. More recently, NWP assimilation systems have played a substantial role in the calibration and validation of new satellite instruments, because of their ability to provide an 'expected value' for every measurement from a new system. Provided the biases in the NWP system can be estimated from other information, statistical analysis of the 'observation minus expected' values can provide rapid identification of anomalies in instrument performance or algorithm performance. The first demonstrations of the value of such diagnostic capabilities were with the scatterometer and altimeter on ERS-1. Recent examples include: the scatterometers and altimeters on ERS-2, ENVISAT and QuickScat, the chemistry instruments on ERS-2 and ENVISAT, the SEVIRI imager on MSG and the AIRS sounder on the AQUA satellite (Cho and Staelin 2006; Divakeria et al. 2006; Fetzer et al. 2006; Strow et al. 2006; Susskind et al. 2006).

An interesting synergy has recently emerged in the interpretation of satellite gravimetric data. The NASA GRACE mission is designed to study temporal changes in the earth's gravity field. As noted by Tapley et al. (2004), success in interpreting GRACE data on secular variations in the gravity field requires great precision in calculating tidal effects in the ocean and then removing those from the GRACE measurements. Oceanographers have long used wind stresses from NWP systems, such as that of ECMWF, to force their ocean models. A novel feature of the gravimetric processing is the additional use of the NWP surface pressure fields to represent atmospheric tidal pressure forcing of ocean tides. Dobslaw and Thomas (2005) use analyses and 3-hour forecasts to address this problem, and suggest that '(ECMWF) forecasts allow one to account for atmospheric variability and corresponding oceanic responses down to semidiurnal timescales, dispensing with any additional model of atmospheric tides'. A general result noted by these authors is the benefit for ocean-tide studies (and thus geodetic studies) of the faithful representation in the NWP pressure analyses of the substantial seasonal and other low-frequency variations in the atmospheric tides.

The synergy between NWP and gravimetric work arises because certain aspects of the high-precision geodetic measurements from the GRACE mission reflect continentalscale fluctuations of soil moisture, a quantity which is of great practical significance and which is otherwise difficult to measure. Andersen et al. (2005) show successful comparisons of inter-annual changes in European summer soil moisture estimated by the GRACE geodetic mission with estimates calculated from the operational ECMWF soilmoisture analysis; while Syed et al. (2005) report successful comparisons of GRACE estimates of fluctuations in water storage in the Amazon basin with similar estimates based on ECMWF analyses. The relevance for GEOSS is that well-validated representations of variations in continental-scale soil moisture and hydrology are valuable for applications of GEOSS interest in areas such as water-resources, energy, climate and agriculture.

\section{(b) Ensemble multi-model seasonal forecasts of crop yields and of malaria incidence}

The World Climate Research Programme's 1985-94 Tropical Ocean-Global Atmosphere (TOGA) initiative developed the observational basis for scientific insight 
into the mechanism of the El Niño Southern Oscillation (ENSO) phenomenon, and inspired the application of coupled atmosphere-land-ocean models in operational multiseasonal forecasting. The main physical basis for predictability on these time-scales is provided by slow atmosphere-ocean interactions in the tropical Pacific. Operational seasonal forecasts with coupled models use ensemble methods to quantify the uncertainties of the forecasts. A consensus of the various forecasts has successfully predicted the major shifts in the ENSO over the last decade.

A feature of seasonal forecasts with coupled models is that time-mean systematic errors in the forecast models can be as large as the signal one is trying to forecast. This has prompted interest in assessing the value of making an ensemble seasonal forecast using several different coupled models. A useful summary of the issues may be found in Palmer (2005), the preface to the special volume of Tellus on the results of an EU-funded experiment (DEMETER*). This experiment used ERA-40 datasets and seven European coupled models in a systematic exploration of multi-seasonal forecast skill in the period 1958-2002. One of the main results of the experiment was a clear confirmation of the skill of multi-seasonal forecasts, and a demonstration of the value of using a multi-model approach to generate the forecasts (Doblas-Reyes et al. 2005; Hagedorn et al. 2005). Prompted by the DEMETER results, a new operational multi-model seasonal forecast system (EUROSIP) was launched in Europe in late 2005, based on ensemble forecasts from three coupled forecast systems (ECMWF, UK Met Office and Météo France) using a common forecast protocol (start dates, duration), with the results archived, verified and displayed in a common format. Research will continue on methods to combine the individual ensembles, as well as on improving the performance of the individual coupled systems and on the means to extract information of direct value to end-users.

Palmer (2005) notes the 'ground-breaking' demonstrations on the benefits of linking the forecast model output to end-user application models, specifically for crop-yield (Cantelaube and Terres 2005; Challinor et al. 2005; Marletto et al. 2005). Each of these studies used the paradigm discussed earlier, by coupling a cascade of general and specialized earth-system models to deliver user-specific forecasts.

A further example of the use of a cascade of models to transform observations into information is provided by the work of Thomson et al. (2006) on early warnings for epidemic malaria in southern Africa. They used a statistical model of malaria occurrence to make hindcasts of annual standardized log malaria incidence over a 20year period based on $\mathrm{CMAP} \dagger$ observed rainfall anomalies for the wet season (November to February) in Botswana (www.cpc.ncep.noaa.gov) and on DEMETER predictions of rainfall anomalies for the same season in Botswana; the DEMETER forecasts are available 4 months earlier than the CMAP observations. Table 1 (from Thomson et al. (2006) where further details may be found) compares ROC scores (relative operating characteristic area, with $100 \%$ indicating a perfect forecast) for the malaria predictions based on the observed (CMAP) rainfall anomalies and on the corresponding forecast anomalies from the DEMETER predictions. The authors comment: "for forecasts of both very low and very high malaria incidence the multi-model DEMETER scores have significantly positive ROC scores. The DEMETER scores are slightly lower than the CMAP scores, but we point out that the DEMETER forecasts are available at least four months before the CMAP data becomes available in early March". The authors also note that "in years in which the forecast probability distribution is different from that

\footnotetext{
* Development of a Multi-model Ensemble system for seasonal to inTERannual prediction.

$\dagger$ Climate Prediction Center Merged Analysis of Precipitation (National Oceanic and Atmospheric Administration).
} 
TABLE 1. RELATIVE OPERATING CHARACTERISTIC (ROC) AREAS FOR ANNUAL STANDARDIZED LOG MALARIA INCIDENCE PREDICTION

\begin{tabular}{lcc}
\hline & \multicolumn{2}{c}{ ROC areas } \\
\cline { 2 - 3 } Event & CMAP $^{1}$ & DEMETER $^{2}$ \\
\hline Very high & $0.93(0.70-1.00)$ & $0.80(0.59-0.96)$ \\
High & $0.36(0.06-0.70)$ & $0.48(0.18-0.79)$ \\
Low & $0.33(0.11-0.58)$ & $0.34(0.12-0.60)$ \\
Very low & $0.91(0.73-1.00)$ & $0.83(0.61-0.99)$ \\
\hline${ }^{1}$ Climate Prediction Center Merged Analysis of Pre- \\
cipitation (NOAA). \\
2DEvelopment of a Multi-model Ensemble system for \\
seasonal to inTERannual prediction. \\
The predictions were carried out using observed \\
CMAP and DEMETER multi-model ensemble pre- \\
cipitation from November to February and have simi- \\
lar forecast quality. CMAP predictions are assessed as \\
probabilistic forecasts with fixed, but unknown uncer- \\
tainty. The 95\% confidence limits obtained by boot- \\
strapping with a sample size of 10 000 are shown \\
in parentheses. (From Thomson et al. (2006) which \\
provides further details.)
\end{tabular}

of climatology, malaria decision-makers can use this information for improved resource allocation".

\section{(c) Developments in forecasting and applications on weekly-monthly time-scales}

Evaluations in the mid-1980s of the promise of forecasts on weekly-monthly timescales were not encouraging. The progress since then in medium-range forecasting, together with the development of successful coupled atmosphere-ocean-land models for multi-seasonal forecasting, prompted a re-evaluation of the prospects for monthly forecasting using coupled models (Vitart 2004). Following the success of experimental tests, ECMWF implemented monthly forecasts as an operational product in October 2004. The new system was discussed by Vitart (2005, personal communication) at a user workshop in June $2005^{*}$. The monthly forecast system creates once a week (starting from 0000 UTC each Thursday) a monthly ensemble forecast (ensemble size 51) with the same ocean model as the seasonal forecast system discussed in subsection 3(b) and with a higher-resolution atmospheric model (T159 v T95). The monthly forecasts are run for 32 days. In Vitart's presentation and in the user evaluations the following definitions are used:

- $\quad$ Week-1 is days 5-11 (Monday to Sunday).

- Week-2 is days 12-18 (Monday to Sunday).

- Week-3 is days 19-25 (Monday to Sunday).

- Week-4 is days 26-32 (Monday to Sunday).

Figure 5 shows an example of the weekly mean forecasts of the anomaly in $2 \mathrm{~m}$ temperature (T2m) for the period 21-27 February 2005, which was anomalously cold over much of western Europe, together with the observed anomaly. The forecasts at week-2 and week-3 for this event look very encouraging. The conclusions of the user workshop (available on the same web-site) indicate that during winter 2004-05 the 

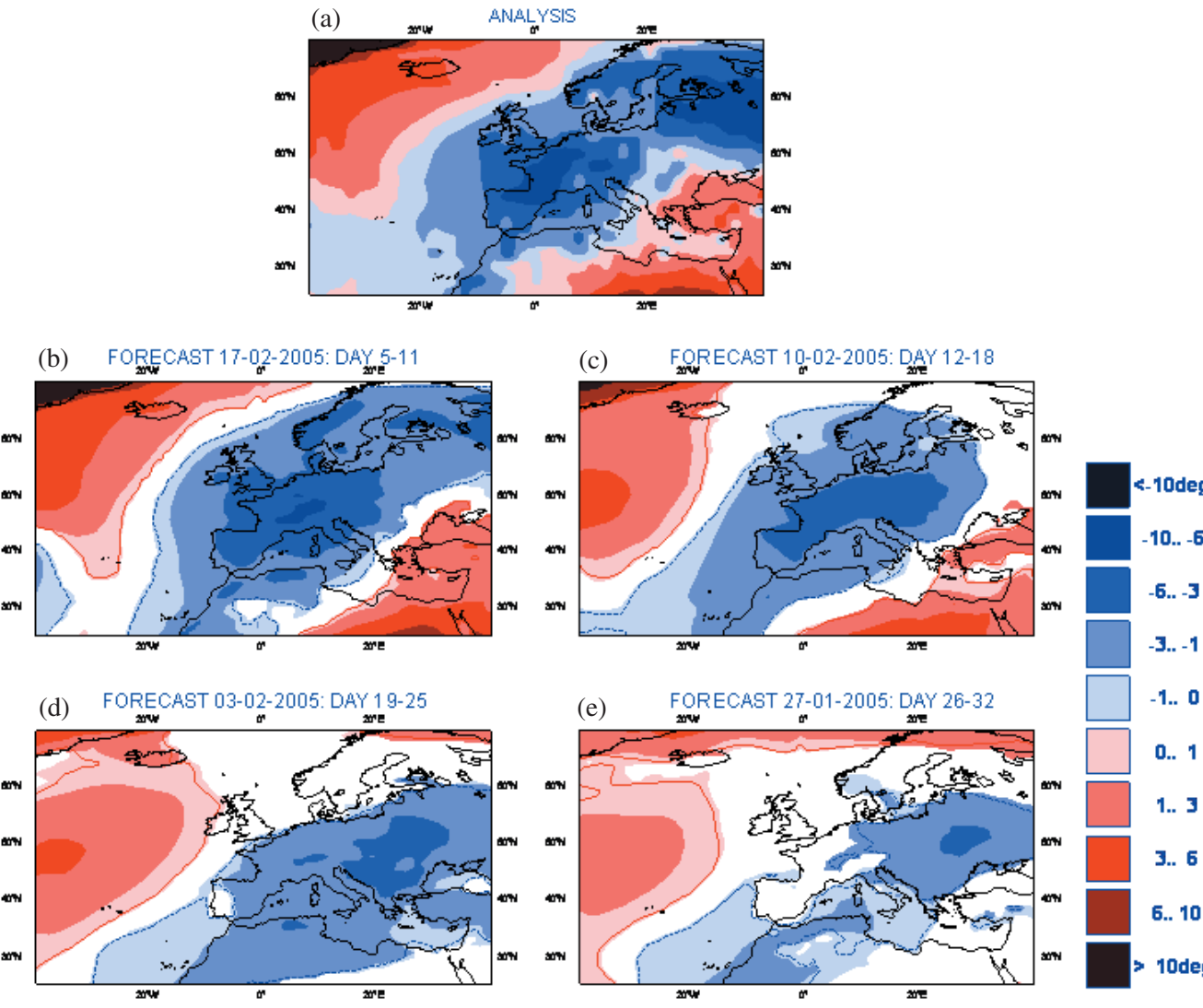

Figure 5. Verification of screen-level temperature $(\mathrm{T} 2 \mathrm{~m})$ forecasts over Europe for the week Monday 21 to Sunday 27 February 2005: (a) analysis; (b) corresponding week-1 (days 5-11) forecast made from 17 February; (c) corresponding week-2 (days 12-18) forecast made from 10 February; (d) corresponding week-3 (days 19-25) forecast made from 3 February; and (e) corresponding week-4 (days 26-32) forecast made from 27 January. The ensemble size is 51; the climate size is 60; shaded areas are above the $90 \%$ significance level; and the solid contour is at $95 \%$ significance. See text for discussion.

system provided good predictions of transitions between flow regimes; the forecasts for weekly-mean T2m for week-2 show skill over persistence of the probabilities of the previous week and, using a similar criterion, show skill to a lesser extent for week-3 and week-4 treated jointly (i.e. days 19-32). Météo France finds moderate skill in T2m forecasts for week-3 over France. In the UK, the Met Office finds that the outer quintiles of the forecast probability distributions for T2m are skilful.

These early operational results are consistent with Vitart's pre-operational results, and give grounds for optimism about future developments. It is still early days with the monthly forecast system, but there are already strong indications that a useful product on major weather shifts in week 2 and possibly in week 3 can be delivered. There is a huge social and economic demand for such products across almost all the GEOSS areas of interest.

Evidence from multi-seasonal forecasts suggests that the forecast errors are dominated by errors in the atmospheric model (D. L. T. Anderson 2005, personal communication). Since the same is probably true to a large extent for the monthly forecast errors, one expects that more and better process-oriented observations will stimulate 
the improved science needed to realize the full potential of both monthly and seasonal forecasts.

\section{(d) Operational earth-system models which encompass atmospheric composition}

As part to the GMES initiative, the EU has funded a new project known as GEMS (Global Environment Monitoring System) using satellite and in situ data to develop a real-time operational assimilation and forecast capability of aerosols, greenhouse gases and reactive gases. The GEMS consortium includes extensive representation of the European science base, with participation of environmental institutes, meteorological services, international agencies and many research institutes. The GEMS operational system will be an extension of current data assimilation and forecast capabilities for NWP with operational implementation planned for 2009. The main users of the GEMS Project will be high-level policy users, operational regional air-quality and environmental forecasters, and the scientific community including GCOS (Global Climate Observing System). Global operational deliverables will include current and forecast threedimensional global distributions (four times daily with a horizontal resolution of 50$100 \mathrm{~km}$, and covering the troposphere and stratosphere) of key atmospheric trace constituents including greenhouse gases, reactive gases and aerosols. The regional objectives of GEMS include operational provision of initial conditions and boundary conditions for operational regional air-quality and 'chemical weather' forecast systems across Europe, following the examples of the Prev'Air system in France and the AirNow system in North America. Part of the motivation for the GEMS project is the wish to provide improved alerts for events such as the 2003 heat waves in western Europe which led to at least 33000 excess deaths (Kosatsky 2005), mostly due to heat stress but partly due to poor air quality.

Satellite data will be a major source of information for the assimilation. The project will develop operational global and regional forecast models, and data assimilation systems to synthesize satellite and in situ data so as to provide initial data for the forecasts. The initial data, being a comprehensive syntheses of many streams of observations, will comprise frequent status-assessments of the atmospheric environment and thus will be invaluable for documenting sources, sinks and transports of atmospheric trace constituents. For validation purposes, the project will generate a retrospective analysis of all accessible remotely sensed data on atmospheric dynamics and composition, as validation material for the ENVISAT-EOS (earth observing system) era (1999-2007). In addition, the project will develop state-of-the-art variational estimates of the sources/sinks, plus inter-continental transports, of many trace gases and aerosols. These estimates will be designed to meet the key information requirements of policy-makers, relevant to the Kyoto and Montreal Protocols, and to the UN Convention on long-range trans-boundary air pollution*.

\section{(e) Prospects for future progress in forecast skill}

The literature reviewed here has presented substantial and sustained progress in improving predictive skill on medium-range time-scales (3-10 days) and on weeklymonthly time-scales. In addition, research has demonstrated the considerable value of multi-seasonal forecasts based on multi-model ensembles. There is abundant evidence of the operation of a virtuous circle between: the sustained availability of earth-system observations, the scientific developments needed to understand and simulate those

\footnotetext{
* Further information is available at http://www.ecmwf.int/research/EU_projects/GEMS/index.jsp
} 
observations, consequential improvements in the quality of forecasts, and the delivery of socio-economic benefits.

Current expectations, based on predictability studies and on analyses of forecast errors, are that there is considerable room for improvement in the earth-system models, and in the starting data for forecasts. Thus, if investments in observation networks, modelling and data assimilation are sustained, then the trends for improved delivery of earth-system forecasts will also be sustained. Direct coupling between earth-system models and end-user application models will increasingly provide effective forecasts of quantities of direct relevance to end-users. We have noted pre-operational examples in ensemble flood forecasts, crop-yield forecasts, and malaria warnings; we also note forthcoming developments for improved air-quality forecasts and related services to the health community.

\section{FUTURE GEOSS REQUIREMENTS FOR OBSERVATIONS}

It is a large and arduous task to prepare and maintain a formal statement of observation requirements - the World Meteorological Organization (WMO) undertakes a substantial biennial survey of its members to update its 'rolling review of requirements'; equally significant efforts have been made by GCOS and by the IGOS_P (Integrated Global Observing Strategy partnership). Here we outline a broad-brush sketch of what the GEOSS observation requirements may be in the coming decades for medium-term and longer predictions of the atmosphere and ocean. The basic requirement is an allweather capability to measure all relevant aspects of the earth-system, where 'relevant' means that the measurements can be used to improve the GEOSS products. The allweather requirement poses a particular challenge because a cloudy atmosphere (found over $50 \%$ of the planet at any time) is opaque to instruments operating at electromagnetic wavelengths shorter than microwave.

For atmospheric dynamics, wind profiles are a prime requirement. Currently the radiosonde network provides all-weather capability but has inadequate coverage. An operational wind lidar in space (following ESA's forthcoming Atmospheric Dynamics Mission) could meet the requirement in clear conditions. This will be supplemented by surface all-weather ocean wind measurements from ships, and from scatterometers on the MetOp series. For vertically resolved atmospheric temperature and humidity profiles, the forthcoming operational advanced infra-red sounders, IASI (Infrared Atmospheric Sounding Instrument) and CrIS, will be valuable in clear-sky conditions. However, the requirement for an all-weather microwave capability delivering comparable vertical resolution poses formidable technical challenges in cloudy areas and especially in rain areas. The best prospects are Global Positioning System (GPS) radio-occultation measurements which operate around $1.4 \mathrm{GHz}$, a frequency range for which a cloudy atmosphere is almost transparent. However, there remains the problem of disentangling the temperature and humidity effects in the lower troposphere. An unexplored aspect of such measurements is the ability of a fleet of GPS receivers in low earth orbit to provide a tomographic profiling capability.

Successful prediction of all elements of the hydrological cycle is a key GEOSS requirement. The use of profiles of rain-rate and clouds (through modelling and assimilation of rain- and cloud-affected satellite measurements in the visible, infra-red and microwave) is still at an early stage of development. In the expectation that the Global Precipitation Mission will fly in the coming decade, it is likely that there will be a requirement in the following decade for an operational follow-on mission. Likewise, successful demonstration of the predictive value of cloud-profile data from missions 
such as CLOUDSAT will surely trigger a requirement for an operational cloud-profiling radar in orbit.

Successful prediction on medium-term and longer time-scales requires much data on ocean circulation and ocean surface conditions. Many instruments can measure seasurface temperature, but there is limited all-weather capability. Microwave imagers, altimeters, scatterometers and synthetic aperture radars provide valuable information on: ocean surface waves; ocean surface stress and winds; ocean-ice extent, age and movement; sea-level height; and ocean surface salinity. A sustained and comprehensive operational observational capability of ocean surface conditions is a key requirement for the coming decades. Because of the opacity of water to electromagnetic radiation, measurement of sub-surface ocean conditions depends on in situ data such as the ARGO floats, and on acoustic remote sensing for ocean tomography. GEOSS will surely generate operational requirements for such observations.

Experience shows that a good 3-day forecast of near-surface temperature in Europe in most seasons cannot be produced without good observations and a good model representation of land-surface and soil processes, and their interactions with the atmosphere. There is evidence that successful longer-term predictions have similar requirements (Ferranti and Viterbo (2006) and references cited therein). Accurate observation of landsurface conditions is essential for many GEOSS predictive purposes, and there are operational requirements for measurements of land-surface skin-temperature, soil moisture, soil type, vegetation, albedo, roughness-length, extent of snow, lake temperatures and ice-cover, atmospheric sources of trace gas and particulates from biomass burning (as well as information on the associated biomass-fuel availability and state of vegetation). In addition, for hydrological purposes there are requirements for in situ and altimeter measurements of the stage of large lakes and rivers.

Use of space-based data for operational assimilation of ozone is already underway at several operational centres. The GEMS project, described in subsection 3(d), will develop a comprehensive operational capability for modelling, monitoring and the assimilation of atmospheric composition as well as dynamics and thermodynamics. Operational data requirements for atmospheric composition will, therefore, include profile measurements of a range of greenhouse gases and reactive gases as well as vertical profiles of a range of aerosol types.

\section{THE RELATIVE COSTS OF EARTH OBSERVATIONS AND INFORMATION PRODUCTS}

Observations represent the major cost element for GEOSS activity. Current costs, based on the budgets of WMO and the Space Agency for earth observations, are estimated to be \$8-10B annually, including in situ data and both operational and research satellites. By comparison, the cost of an operational forecast centre such as ECMWF is about $\$ 40 \mathrm{M}$ annually. The annual cost of ten such centres distributed globally is about $5 \%$ of the cost of observations. An estimate of the cost of the diverse specialized regional and applications activities is not readily available. However, it is unlikely to change the overall indication that observations will dominate the costs of GEOSS. Although we have not discussed the issue here, observing-system experiments to help optimize the observing system will probably be a key feature of the GEOSS scientific activity. There is a long and sustained history of such research since preparations for the Global Weather Experiment in the early and mid 1970s. Such work will need to continue in order to optimize future observing systems.

Assessment of the cost-benefit ratio for different observation strategies is difficult, as it is impossible to carry out a controlled experiment on the atmosphere and society. 
However, for major disasters of the past, a cost-benefit analysis of the value of the observing system of the time, and of today's modelling capabilities, can be facilitated by comparisons of re-analyses and re-forecasts of these occasions with forecasts made at the time. For the re-forecasts of the 1953 Dutch disaster, discussed earlier, it is likely that such a cost-benefit analysis would give a positive outcome. Similar positive outcomes are likely for ongoing re-forecasts of the 1962 storm which cost 200-300 lives in Hamburg (Klinker 2004, personal communication), and the 1966 storm which caused devastating floods in Florence together with an equally devastating storm surge in Venice (R. Buizza and L. Cavaleri 2005, personal communication). An advantage of this approach to value assessment is that earth-system models can be coupled to enduser models (e.g. for floods or surges or health or crop-yield, etc.) so that end-users can make their assessments of value in terms that are directly meaningful to them.

Given the large annual global spend on satellite and in situ observations, governments rightly demand a continually wider range of products derived from their continuing investments in observations. The mission statement from governments to meteorologists and other earth-system scientists is clear: Use every available scientific and technical resource to improve the skill, the scope and the utility of forecasts.

Over the last 15 years, partnerships in Europe have built the first components of an earth information system, addressing the physical and hydrological elements of the atmosphere-ocean-land-surface-ice system, for use in operational forecasting on daily, weekly, and multi-seasonal time-scales; the next step in this phased development will be the extension of current capabilities to encompass atmospheric composition. An integral part of the European developments has been active and continuing dialogue between NWP practitioners and their colleagues in areas such as land-surface, hydrology, ocean waves, oceanography, geodesy, crop forecasting, and more recently environment and health. In many cases this has led to the development of operational and pre-operational ensemble forecast systems, where end-user models are coupled to earth-system ensemble forecasts to produce ensemble forecasts of end-user variables. This work has demonstrated the value of a cascade of earth-system models and data assimilation systems to transform earth-system observations into information of socio-economic value.

Delivery and sustained improvement of the socio-economic benefits depends on sustained investment in observations, and in the science and computing necessary to transform the observations into useful products. An important consideration is that spatial resolution is a key aspect of product accuracy, and improvements in model resolution make heavy demands on computer resources. Given the way computing technology advances ('Moore's Law'), better science and products can continue to be generated provided the GEOSS partners can achieve a sustained budget in real economic terms for observations, science and computing. Since the cost of the investments in science and computing for product generation is substantially smaller than the cost of observations, governments should note that minor variations in the costs of observations represent resources which could have a major impact on the range and quality of the ensuing products.

The benefits of an earth observing system are realized in a wide variety of products, not least of which is science. There is a virtuous circle connecting the provision of sustained observations to the development of new science, and thus to the delivery of improved forecasts. Sustained observations expose our ignorance of important natural processes, and force improvements in the science of forecast models and products. A flow of new science and steadily improving forecasts will provide the motivation to sustain and improve the observational programmes, which are the major cost elements of the forecast process. Given reasonable expectations of technological advances in 
observational instrumentation and in computing, GEOSS can expect to meet its socioeconomic deliverables in a progressive fashion provided governments can sustain, in real economic terms, the current budgets for observations and the necessary scientific computing and operational infrastructure.

\section{ACKNOWLEDGEMENTS}

We are grateful to many colleagues in our respective institutions for illuminating discussions on the issues raised here. Adrian Simmons contributed much to improve the manuscript. A. Hollingsworth was partially supported by contract EU/DG/ENTR/516099; he thanks his colleagues in the ad-hoc GEOSS SubGroup on Data Utilisation, including D. Marbouty (ECMWF), P. Dubreuil (MSC), R. Mailhot (MSC), F. Branksi (NOAA), J. Hill (USGS), M. Gant (NIH), and also R. Anthes (UCAR), for lively debates which clarified many issues.

Andersen, O. B., Seneviratne, S. I., 2005 Hinderer, J. and Viterbo P.

Andersson, E., Bauer, P., Beljaars, A., Chevallier, F., Hólm, E., Janisková, M., Kållberg, Per., Kelly, G., Lopez, P., McNally, A., Moreau, E., Simmons, A. J., Thépaut, J.-N. and Tompkins, A. M.

Arduino, G., Reggiani, P. and Todini, E.

Buizza, R., Houtekamer, P. L., Toth, Z., Pellerin, G., Wei, M. and Zhu, Y.

Cantelaube, P. and Terres, J.-M.

Challinor A. J., Slingo, J. M., Wheeler, T. R. and Doblas-Reyes, F. J.

Cho, C. and Staelin, D. H.

De Roo, A. P. J., Gouweleeuw, B., Thielen, J., Bartholomes. J., Bongioannini-Cerlini, P., Todini, E., Bates, P. D., Horritt, M., Hunter, N., Beven, K., Pappenberger, F., Heise, E., Rivin, G., Hils, M., Hollingsworth, A., Holst, B., Kwaadijk, J., Reggiani, P., van Dijk, M., Sattler, K. and Sprokkereef, E.

Divakaria, M. G., Barnet, C. D., Goldberg, M. D.,

McMillin, L. M., Maddy, E., Wolf, W., Zhou, L. and Liu, X.

Doblas-Reyes F. J., Hagedorn, R. and Palmer, T. N.

Dobslaw, H. and Thomas, M.

\section{REFERENCES}

GRACE-derived terrestrial water storage depletion associated with the 2003 European heat wave. Geophys. Res. Lett., 32, L18405, doi: 10.1029/2005GL023574

2005 Assimilation and modeling of the atmospheric hydrological cycle in the ECMWF Forecasting System. Bull. Am. Meteorol. Soc., 86, 387-402

2005 Recent advances in flood forecasting and flood risk assessment. Hydrol. Earth Syst. Sci., 9, 280-284 (downloadable from http://www.copernicus.org/EGU/hess/9/contents4.htm)

2005 A comparison of the ECMWF, MSC, and NCEP global ensemble prediction systems. Mon. Weather Rev., 133, 1076-1097
2005

2005

2006

2003

2006

2005

2005
Seasonal weather forecasts for crop yield modeling in Europe. Tellus, 57A(3), 476-487

Probabilistic simulations of crop yield over western India using the DEMETER seasonal hindcast ensembles. Tellus, 57A, 498-512

Cloud clearing of Atmospheric Infrared Sounder hyperspectral infrared radiances using stochastic methods. J. Geophys. Res., 111, D9, D09S18, doi: 10.1029/2005JD006013

Development of a European flood forecasting system. Int. J. River Basin Management, 1, 49-59

Validation of Atmospheric Infrared Sounder temperature and water vapour retrievals with matched radiosonde measurements and forecasts. J. Geophys. Res., 111, D9, D09S15, doi: 10.1029/2005JD006116

The rationale behind the success of multi-model ensembles in seasonal forecasting. II: Calibration and combination. Tellus, 57A, 234-252

Atmospheric induced oceanic tides from ECMWF forecasts. Geophys. Res. Lett., 32, L10615, doi: 10.1029/2005GL022990 
Ferranti, L. and Viterbo, P.

Fetzer, E. J.

GEOSS

2004

2005

Gerritsen, H., de Vries, H. and Philippart, M.

Gouweleeuw, B. T., Thielen, J., Franchello, G., De Roo, A. P. J. and Buizza, R.

Hagedorn, R., Doblas-Reyes, F. J. and Palmer, T. N.

Hollingsworth A., Shaw, D. B., Lonnberg, P., Illari, L., Arpe, K. and Simmons, A. J.

Hollingsworth, A., Viterbo, P. and Simmons, A. J.

Kosatsky, T.

McNally, A. P., Watts, P. D., Smith, J. A., Engelen, R., Kelly, G., Thépaut, J. N. and Matricardi, M.

Marletto, V., Zinoni, F., Criscuolo, L., Fontana, G., Marchesi, S., Morgillo, A., Van Soetendael, M., Ceotto, E. and Andersen, U.

Palmer, T.

2005

2005

1986

2004

Pappenberger, F., Beven, K. J., Hunter, N. M., Bates, P. D., Gouweleeuw, B. T., Thielen, J. and de Roo, A. P. J.

Simmons, A. J. and Hollingsworth, A.

Strow, L. L., Hannon, S. E., De-Souza Machado, S., Motteler, H. E. and Tobin, D. C.

Susskind, J., Barnet, C., Blaisdell, J., Iredell, L., Keita, F., Kouvaris, L., Molnar, G. and Chahine, M.

Syed, T. H., Famiglietti, J. S., Chen, J., Rodell, M., Seneviratne, S. I., Viterbo, P. and Wilson, C. R.

Tapley, B. D., Bettadpur, S. and Ries, J. C.

Taylor, J. W. and Buizza, R.

2006

2006 conditions. J. Climate, in press

Validation of Atmospheric Infrared Sounder observations. J. Geophys. Res., 111, D9, D09S01, doi: 10.1029/2005JD007020

GEOSS Framework Document. Downloadable from http://earthobservations.org

GEOSS ten-year implementation plan reference document. GEO 1000R / ESA SP_1284, ISBN 929092986 3. Available from European Space Agency Publications Office, Paris, France

The Dutch continental shelf model. P. 510 in Quantitative skill assessment for coastal ocean models, coastal and estuarine studies. Eds. Daniel Lynch and Alan Davies. Vol. 47, American Geophysical Union, Washington DC, USA

Flood forecasting using medium-range probabilistic weather prediction. Hydrol. Earth Syst. Sci., 9, 365-380 (Downloadable from http://www.copernicus.org/EGU/hess/9/contents4.htm)

The rationale behind the success of multi-model ensembles in seasonal forecasting. I: Basic concept. Tellus, 57A, 219-233

Monitoring of observation and analysis quality by a data assimilation system. Mon. Weather Rev., 114, 861-879

The relevance of numerical weather prediction for forecasting natural hazards and for monitoring the global environment. In Met Monograph: A half century of progress in meteorology: A tribute to Richard Reed. Eds. R. H. Johnson and R. A. Houze Jr. American Meteorological Society, Boston, USA
2005

2006

2005

2005

2005

2002

2006

2006

2005
The 2003 European heat waves. Euro. Surveill., 10(7), 148-149 (Downloadable from www.eurosurveillance.org/em/v10n07/1007-222.asp)

The assimilation of AIRS radiance data at ECMWF. Q. J. $R$. Meteorol. Soc., 132, 935-957

Evaluation of downscaled DEMETER multi-model ensemble seasonal hindcasts in a northern Italy location by means of a model of wheat growth and soil water balance. Tellus, $\mathbf{5 7 A}$, 488-497

Development of a European Multimodel Ensemble system for seasonal to inTERannual prediction. Tellus , 57A, 217-218

Cascading model uncertainty from medium range weather forecasts (10 days) through a rainfall-runoff model to flood inundation predictions within the European Flood Forecasting System (EFFS). Hydrol. Earth Syst. Sci., 9, 381-393 (Downloadable from http://www.copernicus.org/EGU/hess/9/contents4.htm)

Some aspects of the improvement in skill of numerical weather prediction. Q. J. R. Meteorol. Soc., 128, 647-677

Validation of the Atmospheric Infrared Sounder radiative transfer algorithm. J. Geophys. Res., 111, D9, D09S06, doi: 10.1029/2005JD006146

Accuracy of geophysical parameters derived from Atmospheric Infrared Sounder/Advanced Microwave Sounding Unit as a function of fractional cloud cover. J. Geophys. Res., 111, D9, D09S17, doi: 10.1029/2005JD006272

Total basin discharge for the Amazon and Mississippi river basins from GRACE and a land-atmosphere water balance. Geophys. Res. Lett., 32, L24404, doi: 10.1029/2005GL024851

GRACE measurements of mass variability in the earth system. Science, 305, 503-505

Using weather ensemble predictions in electricity demand forecasting. Int. J. Forecasting, 19, 57-70 
Thomson, M. C.,

Doblas-Reyes, F. J.,

Mason, S. J., Hagedorn, R.,

Morse, A. P. and Palmer, T. N.

Vitart, F.

Uppala, S. M., Kållberg, P. W., Simmons, A. J., Andrae, U., da Costa Bechtold, V.,

Fiorino, M., Gibson, J. K., Haseler, J., Hernandez, A., Kelly, G. A., Li, X., Onogi, K., Saarinen, S., Sokka, N.,

Allan, R. P., Andersson, E., Arpe, K., Balmaseda, M. A., Beljaars, A. C. M., van de Berg, L., Bidlot, J.,

Bormann, N., Caires, S., Chevallier, F., Dethof, A., Dragosavac, M., Fisher, M., Fuentes, M., Hagemann, S., Hólm, E., Hoskins, B. J.,

Isaksen, L.,

Janssen, P. A. E. M., Jenne, R., McNally, A. P., Mahfouf, J.-F., Morcrette, J.-J., Rayner, N. A., Saunders, R. W., Simon, P., Sterl, A., Trenberth, K. E., Untch, A., Vasiljevic, D., Vitart, F.
2006 Malaria early warnings based on seasonal climate forecasts from multi-model ensembles. Nature, 439, 576-579

2004 Monthly forecasting at ECMWF. Mon. Weather Rev., 132, 27612779

2005 The ERA-40 Reanalysis. Q. J. R. Meteorol. Soc., 131, 2961-3012, doi: $10.1256 /$ qj.04.176 2779, doi: 10.1175/MWR2826.1 